\title{
Technè
}

La science au service de l'histoire de l'art et de la préservation des biens culturels

$42 \mid 2015$

Science et conservation

\section{Développement d'une nouvelle méthode de désinsectisation sous vide partiel des objets $d u$ patrimoine en bois}

Development of a new method of partial vacuum treatment for the disinsectization of wooden objects belonging to cultural heritage

Loïc Caillat, Gilles Chaumat, Lionel Blanc et Thomas Guiblain

\section{OpenEdition}

Journals

Édition électronique

URL : http://journals.openedition.org/techne/7350

DOI : $10.4000 /$ techne.7350

ISSN : 2534-5168

Éditeur

C2RMF

Édition imprimée

Date de publication : 1 décembre 2015

Pagination : 121-126

ISBN : 978-2-7118-6249-8

ISSN : $1254-7867$

\section{Référence électronique}

Loïc Caillat, Gilles Chaumat, Lionel Blanc et Thomas Guiblain, « Développement d'une nouvelle

méthode de désinsectisation sous vide partiel des objets du patrimoine en bois », Technè [En ligne],

42 | 2015, mis en ligne le 01 décembre 2015, consulté le 11 mars 2021. URL : http://

journals.openedition.org/techne/7350; DOI : https://doi.org/10.4000/techne.7350

\section{(ब) $\odot \Theta$}

La revue Technè. La science au service de l'histoire de l'art et de la préservation des biens culturels est mise à disposition selon les termes de la Licence Creative Commons Attribution - Pas d'Utilisation Commerciale - Pas de Modification 4.0 International. 
Loïc Caillat

Gilles Chaumat

Lionel Blanc

Thomas Guiblain

\section{Développement d'une nouvelle méthode de désinsectisation sous vide partiel des objets du patrimoine en bois}

Résumé. ARC-Nucléart a entrepris de mettre au point un procédé original de désinsectisation sous vide partiel, efficace à la fois pour les larves et les oufs d'insectes. Le principe de ce traitement est de réaliser un vide partiel autour des objets pour dessécher les insectes (eau libre) sans dessécher le bois (eau liée). Le dispositif est principalement constitué d'une enceinte sous vide partiel (inférieur à 100 mbar) permettant de contrôler l'humidité ambiante (entre 40 et $60 \%$ HR). Une importante campagne d'essais a été effectuée pour valider le procédé avec plus de deux mille plaquettes de différentes essences de bois moderne, avec ou sans polychromie. L'étude réalisée a permis de démontrer qu'il était possible de mettre en cuvre un traitement à basse pression (50 mbar), à température ambiante (entre 20 et $25^{\circ} \mathrm{C}$ ), pour désinsectiser des objets en bois rapidement (quelques jours) avec une efficacité satisfaisante. Le deuxième constat important de ces travaux est qu'un traitement à basse pression a permis de respecter les polychromies des objets lorsque l'humidité dans l'enceinte était régulée.

Mots-clés. Désinsectisation, anoxie, traitement sous vide partiel.
Development of a new method of partial vacuum treatment for the disinsectization of wooden objects belonging to cultural heritage

\begin{abstract}
ARC-Nucléart has undertaken to develop a new disinsectization process, which destroys both the larvae and eggs of insects under partial vacuum (small injections of nitrogen gas). The basic principle of this treatment is to create a partial vacuum around the objects in order to dry the insects (free water) without drying the wood (bound water). The device is made up of a partial vacuum chamber (pressure lower than 100 mbar) enabling us to control the ambient humidity (between $40 \%$ and $60 \%$ RH). A large number of tests were carried out to validate the process on over 2,000 samples of different species of modern wood, with or without polychromy. This study enabled us to show that it was possible to employ a low-pressure treatment (50 mbar), at an ambient temperature (between $20^{\circ} \mathrm{C}$ and $25^{\circ} \mathrm{C}$ ), to destroy insects from wooden objects rapidly (a few days) with satisfactory results. The second important outcome of these experiments was that lowpressure treatment did not impair the polychromy of wooden objects when the humidity in the chamber was regulated.
\end{abstract}

Keywords. Disinsectization, anoxia, partial vacuum treatment.

\section{Introduction}

Actuellement, différentes techniques sont disponibles pour désinsectiser les collections patrimoniales en bois. En dehors de la fumigation à l'oxyde d'éthylène ou avec d'autres produits chimiques toxiques, soumis à une réglementation de plus en plus contraignante, deux procédés curatifs sont aujourd'hui très couramment utilisés : l'anoxie statique ou dynamique et l'irradiation gamma ${ }^{1}$. Nous pouvons citer aussi la congélation qui apparaît comme une technique très prometteuse, mais qui n'est pas encore proposée aux objets avec polychromie ${ }^{2}$.

Bien que l'anoxie dynamique soit considérée comme le traitement de désinsectisation de référence par les ateliers spécialisés, elle présente au moins deux inconvénients majeurs. La durée de traitement est au minimum de vingt et un jours, pendant lesquels il faut maîtriser parfaitement les paramètres environnementaux : le taux d'oxygène, la température et l'hygrométrie. Par ailleurs, la fiabilité du protocole n'est pas garantie sur les objets particulièrement massifs (plusieurs centimètres d'épaisseur), puisque la désorption de l'oxygène dans l'azote est progressive et limitée par l'effet de confinement du bois.

L'atelier ARC-Nucléart utilise avec succès depuis plus de quarante ans le rayonnement gamma pour désinsectiser les objets en bois qui lui sont confiés. Néanmoins, depuis 2008, il a développé un procédé de désinsectisation original pour proposer une alternative au traitement des objets non démontables qui comporteraient des matériaux sensibles aux rayonnements ionisants, comme par exemple les matériaux transparents. Cette nouvelle technique peut s'apparenter à une variante du procédé par anoxie dynamique, en remplaçant le balayage d'azote à pression atmosphérique par un très faible balayage d'azote sous vide partiel ( $<100 \mathrm{mbar})$. Cet article fait la synthèse des résultats issus de plusieurs campagnes d'essais à partir de milliers d'échantillons en bois, polychromés ou non, afin de vérifier que les niveaux 
d'innocuité de la méthode sur ces matériaux sont comparables à ceux rencontrés lors d'un traitement par anoxie.

\section{Le principe du procédé}

Lors d'un traitement par anoxie, se pose le problème du piégeage de l'air résiduel dans la porosité du bois. Nous sommes donc partis du principe qu'il était plus aisé d'utiliser une dépression (aspiration) pour éliminer efficacement l'air contenu dans les matériaux poreux que d'effectuer un balayage de gaz neutre autour des objets. Puis nous avons envisagé d'utiliser une basse pression pour tuer directement les larves et les œufs d'insectes xylophages par déshydratation au lieu de les asphyxier principalement comme c'est le cas dans les traitements d'anoxie.

L'utilisation du vide partiel ou de la basse pression comme moyen de désinsectisation relève de deux constats :

- les insectes ont besoin d'oxygène et d'eau pour vivre. Comme tous les arthropodes, ils ne possèdent pas de squelette interne, mais un exosquelette (une cuticule externe de chitine molle pour la larve) qui leur permet de limiter les pertes d'eau en milieu aérien. La respiration se fait grâce à des trachées qui constituent un réseau apportant l'oxygène directement aux cellules. Ces trachées s'ouvrent sur l'extérieur par des stigmates respiratoires à ouverture variable, répartis sur le corps de l'insecte ${ }^{4}$;

- si nous comparons la nature de l'eau présente dans un objet en bois dit «sec » (environ $14 \%$ d'eau dans un air à $50 \%$ d'hygrométrie) à celle d'un insecte, on note que, dans le premier cas, l'eau est intimement « liée » par liaison hydrogène aux molécules de cellulose et d'hémicellulose du bois, tandis que l'eau interne existant dans les liquides vitaux de l'insecte (l'hémolymphe) se présente sous forme d'eau « libre » à l'état liquide ${ }^{3}$.

Dans un environnement où règne une pression très inférieure à la pression atmosphérique, les quantités d'oxygène et d'eau disponibles diminuent. Dans de telles conditions, l'eau liquide se transforme plus facilement en vapeur d'eau. L'eau sous forme liquide contenue dans le corps des larves et des oufs d'insectes va, du fait des basses pressions, se vaporiser, ce qui n'est pas le cas de l'eau liée qui reste adsorbée dans les fibres du bois et dont le départ nécessite davantage d'énergie pour rompre les liaisons hydrogènes entre l'eau et le bois.

À basse pression, le mécanisme respiratoire des insectes accentue le phénomène de dessèchement. En temps normal, les pores (ou stigmates) respiratoires disséminés sur tout le corps de la larve d'insecte restent ouverts peu de temps pour limiter les déperditions en $\mathrm{eau}^{3}$. À basse pression, ces mêmes orifices vont rester ouverts plus longtemps, probablement en raison du manque d'oxygène ${ }^{3}$. Le milieu intérieur de l'insecte, constitué majoritairement par l'hémolymphe formée de 85 à $90 \%$ d'eau, se déshydrate rapidement. Comme pour les adultes, les œufs subissent également une dessiccation à travers leur membrane, conduisant à leur mort accélérée si le niveau de vide est suffisamment important. Dans un traitement sous vide partiel, la mortalité ne survient pas directement du fait d'un manque d'oxygène, mais plutôt d'une déshydratation forcée des larves d'insectes ou de leurs œufs. Il ne devrait pas y avoir de risque de déformation du bois si l'on veille à maintenir, dans l'atmosphère résiduelle de l'enceinte, une valeur d'hygrométrie constante proche de celle de l'environnement d'origine de l'objet dans lequel il était en équilibre.

\section{Description de l'enceinte de traitement expérimental}

Le procédé de désinsectisation à basse pression devrait permettre une désinsectisation plus rapide que l'anoxie et de manière plus fiable sans utiliser de gaz toxiques, sans interaction avec la matière, et sans contrainte mécanique sur les objets. Le principal obstacle à la mise en œuvre de cette méthode est le risque de décollement des couches très fragiles de polychromie sans système de régulation de l'hygrométrie : soit l'atmosphère est trop sèche et le bois se rétracte, soit elle est trop humide et le bois gonfle. Ces déformations du bois sur lequel repose la polychromie pourraient conduire au décollement et l'altération de celle-ci. Afin d'éviter ces déformations, nous avons mis au point un dispositif de régulation pour stabiliser l'hygrométrie pendant toute la durée du traitement, indépendamment des basses pressions établies dans l'enceinte de traitement. Dans le dispositif développé, l'humidité est régulée par une entrée d'azote humide.

Les essais effectués ont permis de montrer qu'il était possible de maintenir une humidité relative autour de 50 à $70 \%$, même à basse pression. Des tests ont été effectués

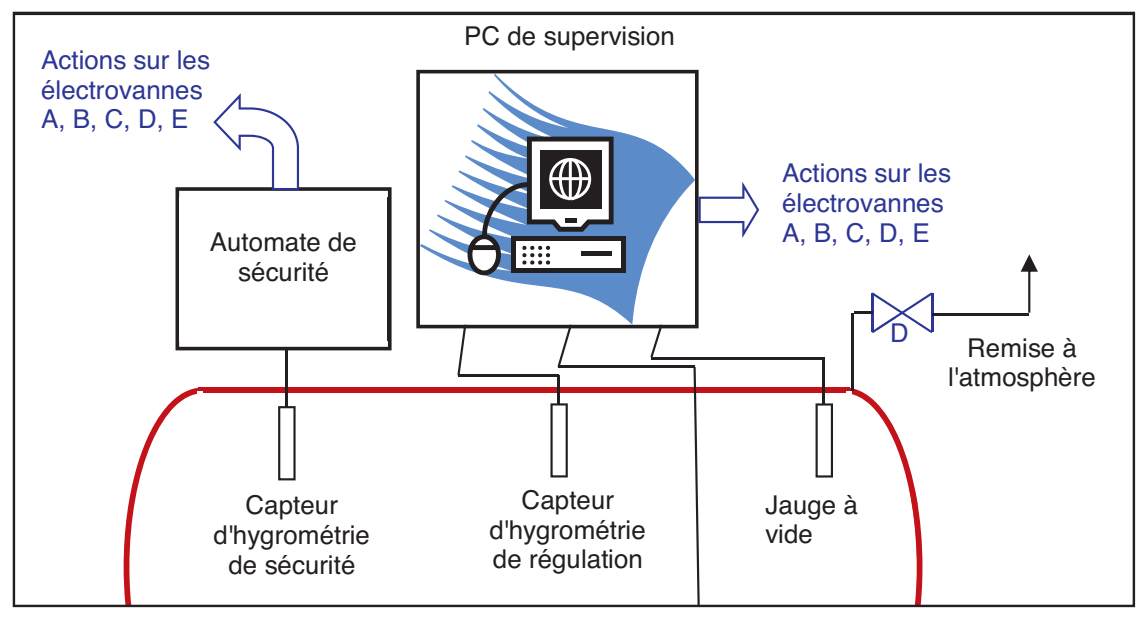

Fig. 1. Synoptique du fonctionnement détaillé de l'enceinte. 


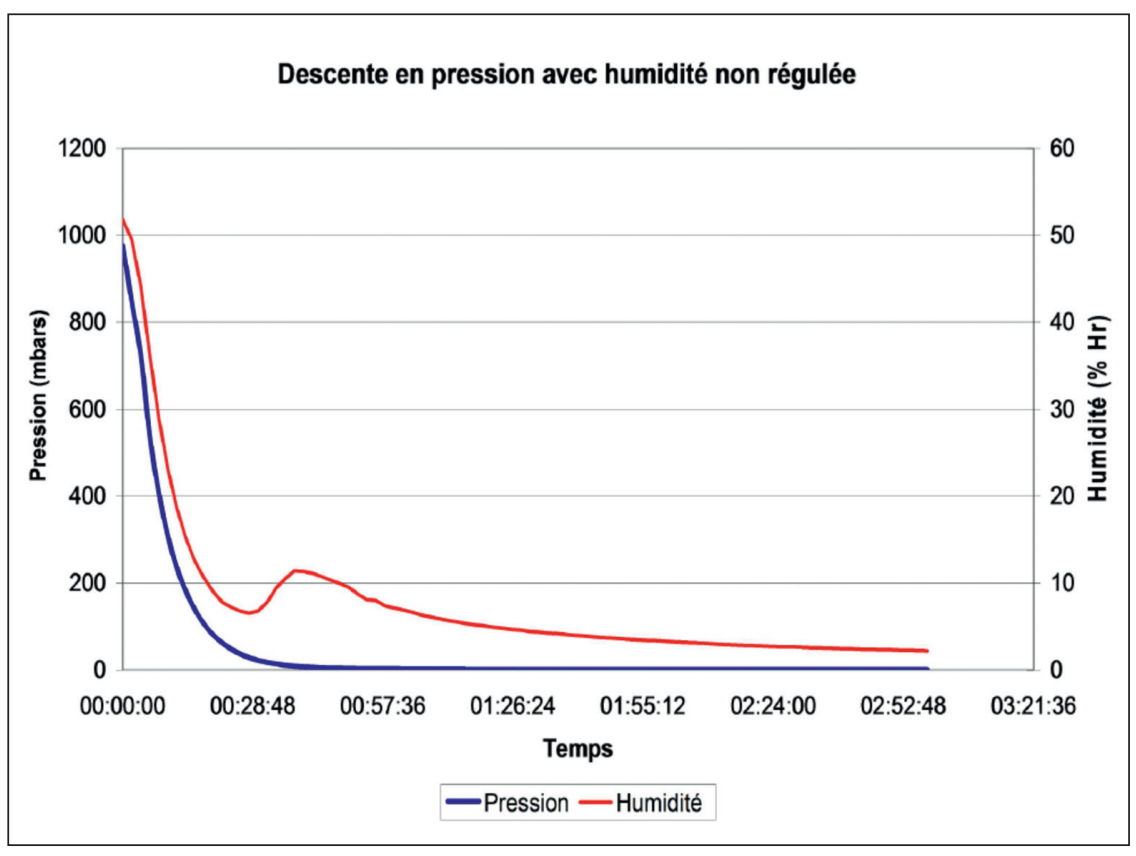

Fig. 2. Courbes de la pression (bleue) et de l'hygrométrie (rouge) lors d'une baisse de pression dans l'enceinte de traitement sans régulation de l'hygrométrie.

jusqu'à 30 mbar, et cela pendant toute la durée d'un traitement sur plusieurs jours. Le dispositif est constitué d'une enceinte cylindrique en acier inox d'un volume de $2,8 \mathrm{~m}^{3}$, équipée de tous les éléments nécessaires pour mesurer en continu la température, l'humidité et la pression de l'atmosphère résiduelle. Le pilotage du cycle est effectué par l'intermédiaire d'un système informatique de supervision sous programmation LABVIEW. Des écrans avec des synoptiques sont utilisés pour diriger les différents organes du dispositif. Toutes les données sont enregistrées automatiquement afin de conserver une traçabilité de tous les cycles effectués. De nombreux paramètres sont réglables tels que les consignes d'humidité ou de

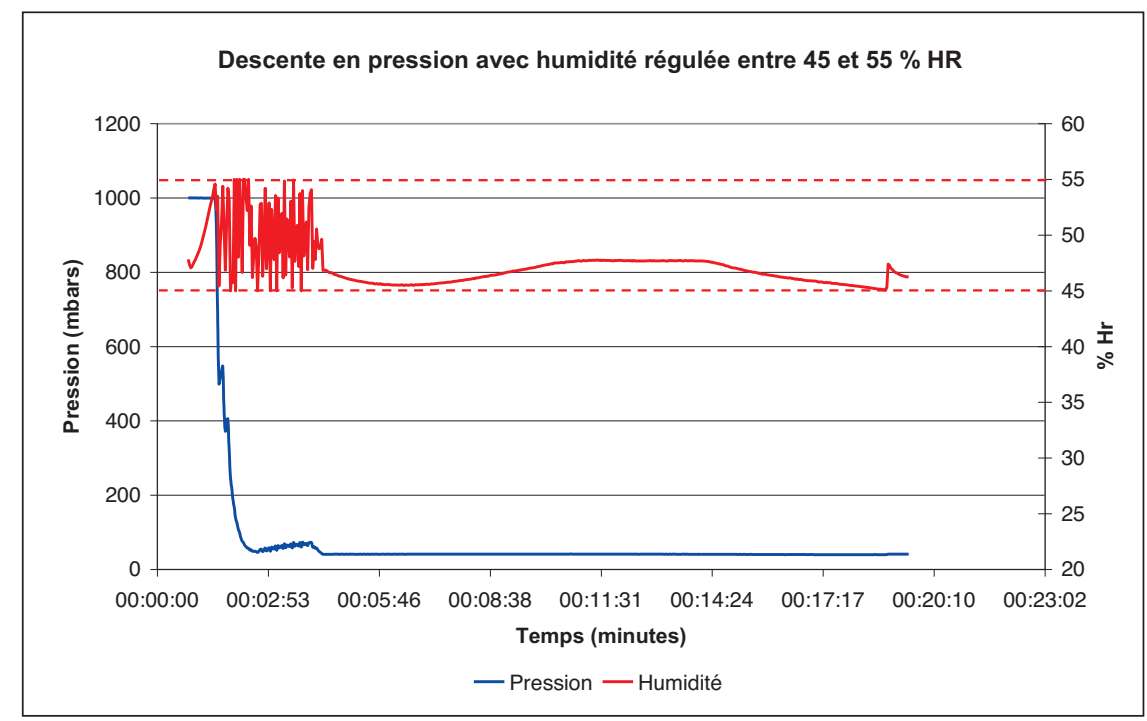

Fig. 3. Courbes de la pression (bleue) et de l'hygrométrie (rouge) lors d'une baisse de pression dans l'enceinte de traitement avec régulation hygrométrique. pression, la durée des cycles de désinsectisation, l'activation des alarmes, etc. (fig. 1). Le pilotage d'un cycle consiste à réguler en parallèle deux paramètres par le dispositif de supervision : la pression et l'humidité relative. La pression est gérée au moyen d'une pompe à vide selon les indications données par un capteur de pression, tandis que l'humidité dans l'enceinte est contrôlée par le dispositif qui pilote l'hydratation. Pour améliorer la diffusion de l'humidité dans l'enceinte, un système de buses (injection multipoints) a été ajouté pour éviter une surhydratation localisée au point d'entrée.

Afin de sécuriser le dispositif, une deuxième sonde d'humidité, indépendante du système de supervision, permet d'arrêter le cycle et de remettre l'enceinte à la pression atmosphérique en cas d'urgence, si jamais une dérive d'humidité réelle est constatée malgré la régulation active de l'humidité. Nous avons relevé l'évolution de l'hygrométrie en fonction de la pression de l'enceinte (fig. 2). On s'aperçoit qu'à 800 mbar, l'hygrométrie « libre » (sans régulation) descend au niveau du seuil critique de $40 \%$ pouvant altérer certains objets fragiles, jusqu'à atteindre finalement $10 \%$ HR à 100 mbar ; ce qui est beaucoup trop bas et dangereux pour les objets polychromés. Lorsque le système d'humidification par injection d'azote chargé en humidité est activé, il est possible de garantir un taux d'humidité, compris dans des plages préréglées, pour des pressions descendant jusqu'à 30 mbar avec une température d'environ $25^{\circ} \mathrm{C}$. L'humidité relative du milieu peut par exemple être régulée à des valeurs comprises entre 45 et $55 \%$ HR (fig. 3) à l'aide d'un dispositif de micro-bullage d'azote à travers une réserve d'eau chauffée à $60^{\circ} \mathrm{C}$.

\section{Mise au point du protocole de désinsectisation}

Pour définir les conditions d'utilisation de basses pressions comme moyen de désinsectisation des objets du patrimoine, il est nécessaire de répondre à deux objectifs :

- tout d'abord préciser les conditions pour lesquelles la destruction des larves et des œufs d'insectes est effective en utilisant un balayage d'azote sous vide partiel ; 
- ensuite, une fois un protocole établi, s'assurer de l'innocuité de la méthode sur les objets soumis au traitement, notamment les bois polychromés.

Pour déterminer le protocole de traitement, nous avons effectué des essais de mortalité en exposant des insectes à différents niveaux de basses pressions. Le choix de l'insecte modèle s'est porté sur le capricorne des maisons, Hydrolupes bajalus, car il est décrit par des études menées au laboratoire du musée du quai Branly comme l'un des plus résistants à l'anoxie ${ }^{1}$. Par ailleurs, il est facile de s'en procurer et la grande taille des larves rend leur manipulation très aisée. La dépression a été obtenue par simple aspiration de l'air contenu dans l'enceinte. Les pressions testées étaient successivement de 30, 50, 80 et 100 mbar. Lors de ces essais, nous avons choisi de travailler avec une valeur constante de l'hygrométrie proche de $50 \%$, car cette valeur médiane est représentative de l'environnement des objets en bois sec conservés à l'intérieur de bâtiments.

L'observation des larves d'insectes après traitement montre une forte déshydratation conduisant à une perte significative de volume et un durcissement des tissus (plus ou moins importants selon la valeur de la consigne d'humidité fixée pour le traitement) indiquant la mortalité des sujets.
Par ailleurs, après quelques heures seulement à l'air, les larves ont bruni, signe d'une oxydation des tissus par l'oxygène de l'air, indiquant une absence d'activité métabolique, ce qui confirme la mort des larves et des œufs (fig. 4). Les meilleurs résultats ont été obtenus avec $50 \%$ d'hygrométrie à $20^{\circ} \mathrm{C}$ et une durée d'exposition de cinqjours à 50 mbar. Ces paramètres constitueront désormais notre protocole de référence.

\section{Expérimentations sur un échantillonnage de plaquettes de bois polychromées}

Pour valider l'innocuité de cette méthode sur les polychromies, des plaquettes rectangulaires modèles, de format 104 x 61 x $10 \mathrm{~mm}$, ont été réalisées dans différentes essences de bois (chêne, noyer, hêtre, châtaignier, sapin, tilleul), avec deux types de sous-couches $\left(\mathrm{CaCO}_{3}\right.$ : blanc de Meudon/colle de peau; $\mathrm{CaSO}_{4}$ : blanc de Bologne/colle de peau) et dix types de polychromies. Pour la couche picturale, nous avons utilisé quatre types de liants associés aux différents pigments (peinture au blanc d'œuf, jaune d'œuf, œuf entier et peinture à l'huile). Les objets polychromés ont été réalisés en enduisant les plaquettes de trois couches de blanc, puis de deux couches

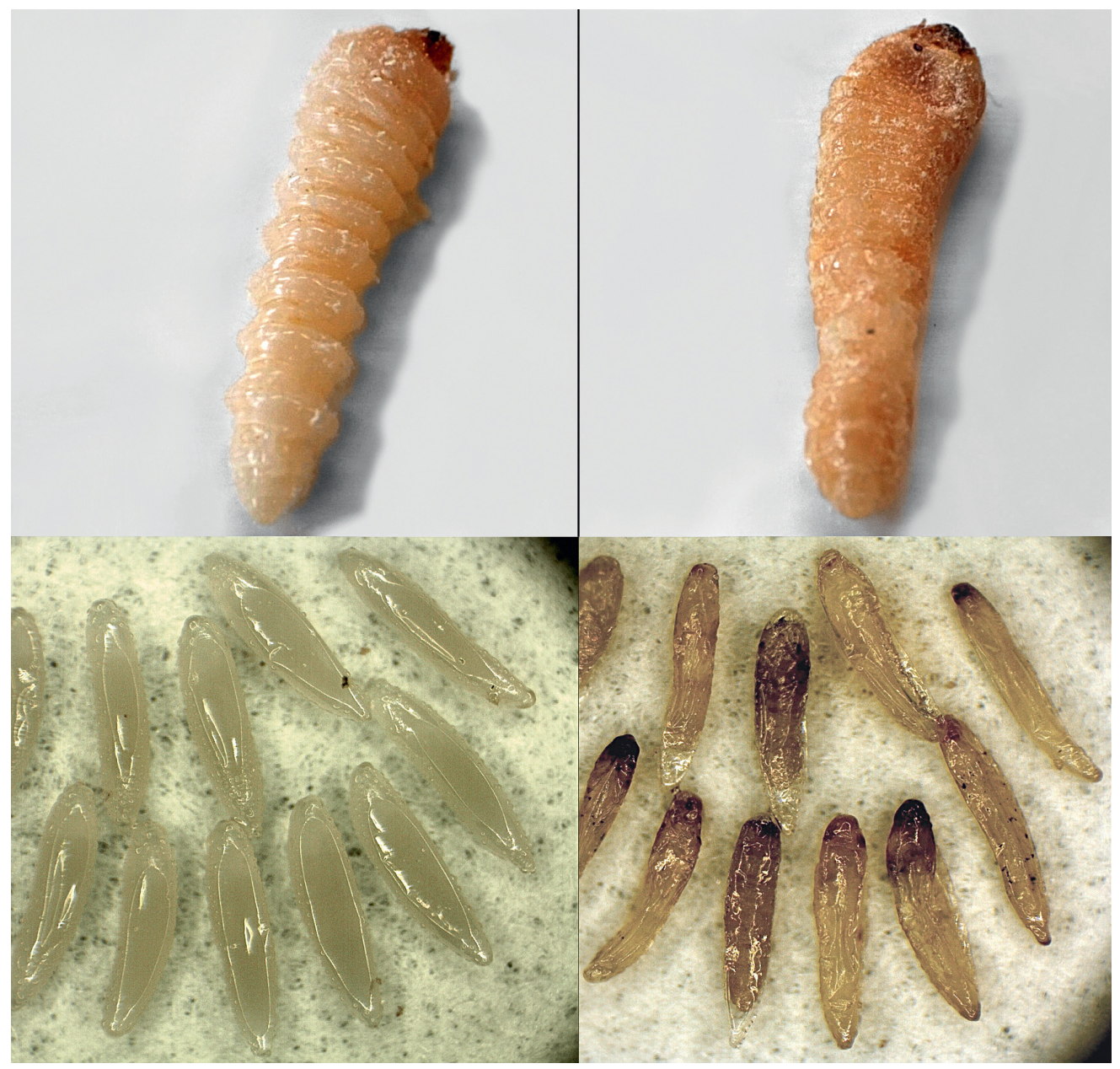

Fig. 4. Larves (en haut) et œufs (en bas) de capricorne des maisons avant (à gauche) et après (à droite) traitement de désinsectisation à 50 mbar. (c) ARC-Nucléart. 
de polychromie. Pour chaque polychromie, un échantillonnage statistique de vingt plaquettes a été utilisé pour chacune des six essences, soit un total de plus de deux mille quatre cents plaquettes.

Une fois les échantillons réalisés, nous avons relevé leurs dimensions, leur masse et leur couleur afin d'en caractériser l'état initial. Nous les avons également photographiés pour suivre leur état de surface avant et après le passage dans l'enceinte de traitement. Les mesures de colorimétrie ont été prises avec un spectrophotomètre qui donne les coordonnées de la couleur mesurée dans un espace de couleur appelé CIELAB avec les coordonnées L*a*b*. Pour évaluer un changement de couleur durant l'anoxie, on calcule $\Delta \mathrm{E}$ qui correspond à la distance entre deux couleurs placées dans cet espace de couleur. Un observateur peu entraîné peut distinguer facilement un $\Delta \mathrm{E}=5$ entre deux couleurs. Un professionnel bien entraîné est capable de discerner, en moyenne, un $\Delta \mathrm{E}$ compris entre 1 et 2 .

Durant la première campagne d'essais, les échantillons de bois ont été exposés à une atmosphère à basse pression (30 mbar) sans régulation d'hygrométrie (protocole dégradé). Dans les résultats qui suivent, nous avons observé le comportement du bois polychromé dans les conditions décrites cidessus, quand il est nu ou avec une sous-couche. En l'absence de régulation de l'hygrométrie, les plaquettes de bois subissent de très légères déformations (tuilage), accompagnées de pertes de masses mesurables allant jusqu'à $2 \%$ pour le sapin. On observe que ces pertes de masse varient de manière significative en fonction de l'essence considérée et de la présence ou non de la sous-couche. Sans sous-couche picturale, le bois se déshydrate davantage pour chacune des essences considérées. Sur le plan des variations dimensionnelles, celles-ci restent inférieures à 1,5\% de déformé (fig. 5), quelle que soit la direction considérée (radiale, tangentielle ou longitudinale).

Il semble donc, au vu de ces résultats, qu'une basse pression (30 mbar) ne présente pas de risque majeur pour les polychromies (décollement des polychromies du substrat en bois), même sans régulation hygrométrique. Il apparaît que la présence d'une sous-couche améliore la protection des polychromies en limitant les pertes en eau et les déformations par rapport au bois non recouvert. Dans la deuxième série d'expérimentations, le protocole impose une hygrométrie régulée à $50 \%$ pendant cinq jours dans une atmosphère à

\begin{tabular}{|l|c|c|c|c|}
\hline Polychromies & Chêne & Noyer & Tilleul \\
\hline Sous-couche au blanc de Meudon & \multicolumn{3}{|c|}{ Variation massique } \\
\hline Laque de garance/jaune d'œuf & $+0,11$ & $+0,26$ & $+0,20$ \\
\hline Vermillon/blanc d'œuf & $-0,30$ & $-0,38$ & $-0,45$ \\
\hline Malachite/œuf entier & $+0,02$ & $+0,31$ & $+0,22$ \\
\hline Azurite/huile & $-0,44$ & $+0,11$ & $+0,05$ \\
\hline Bleu de Prusse/huile & $+0,06$ & $+0,18$ & $-0,69$ \\
\hline Sans & $+0,21$ & $+0,43$ & $+0,22$ \\
\hline Sous-couche au blanc de Bologne & Variation massique \\
\hline Laque de garance/jaune d'œuf & $-0,21$ & $-0,28$ & $-0,21$ \\
\hline Vermillon/blanc d'œuf & $+0,50$ & $-0,89$ & $-0,76$ \\
\hline Malachite/ œuf entier & $-0,16$ & $-0,22$ & $-0,11$ \\
\hline Azurite/huile & $-0,54$ & $-0,71$ & $-0,64$ \\
\hline Bleu de Prusse/huile & $-0,52$ & $-0,66$ & $-0,62$ \\
\hline Sans & $-0,08$ & $-0,26$ & $-0,21$ \\
\hline
\end{tabular}

Fig. 6. Variation de masse (\%) d'une série de plaquettes polychromées, avec sous-couche au blanc de Meudon ou blanc de Bologne et colle de peau, exposées durant 5 jours à 50 mbar et à une hygrométrie de $50 \%$.

50 mbar. Les variations de masse sont dans tous les cas très faibles, elles ne dépassent pas les $0,5 \%$ en prise de masse et $1,2 \%$ en perte de masse (fig. 6). Ces résultats démontrent l'intérêt de la régulation hygrométrique qui permet de limiter les variations massiques du bois. Les écarts dimensionnels peuvent être considérés comme négligeables, compte-tenu du fait que les fluctuations observées correspondent aux incertitudes de mesure et qu'elles ne s'accompagnent d'aucune altération visible des polychromies : pas de fissures, ni de déformations des plaquettes. Les résultats de colorimétrie sont en moyenne satisfaisants, les valeurs de $\Delta \mathrm{E}$ inférieures à 2. Par conséquent, nous pouvons conclure que le traitement de désinsectisation à basse pression régulé en hygrométrie ne semble pas altérer la couleur des polychromies.

\begin{tabular}{|l|c|c|c|c|c|c|c|c|}
\hline \multirow{2}{*}{ Essences } & \multicolumn{4}{|c|}{ Bois nu } & \multicolumn{4}{c|}{ Avec blanc de Meudon } \\
\cline { 2 - 9 } & Longueur & Largeur & Épaisseur & Masse & Longueur & Largeur & Épaisseur & Masse \\
\hline Sapin & $-0,85 \%$ & $-0,07 \%$ & $-1,16 \%$ & $-2,06 \%$ & $-0,36 \%$ & $-0,08 \%$ & $-0,47 \%$ & $-1,15 \%$ \\
\hline Chêne & $-0,70 \%$ & $-0,06 \%$ & $-0,58 \%$ & $-1,69 \%$ & $-0,37 \%$ & $-0,06 \%$ & $-0,36 \%$ & $-0,95 \%$ \\
\hline Noyer & $-1,23 \%$ & $-0,01 \%$ & $-0,77 \%$ & $-1,86 \%$ & $-0,44 \%$ & $-0,10 \%$ & $-0,39 \%$ & $-1,15 \%$ \\
\hline Hêtre & $-0,49 \%$ & $-0,02 \%$ & $-0,49 \%$ & $-1,64 \%$ & $-0,57 \%$ & $-0,10 \%$ & $-0,44 \%$ & $-1,14 \%$ \\
\hline
\end{tabular}

Fig. 5. Variations dimensionnelles et massiques des échantillons traités à 30 mbar sans régulation hygrométrique. 


\section{Conclusion et perspectives}

La présente étude a permis de montrer qu'il était tout à fait possible d'utiliser un traitement à basse pression à 50 mbar avec une température comprise entre 20 et $25^{\circ} \mathrm{C}$ pour désinsectiser des objets en bois avec une durée plus courte et une meilleure fiabilité que les traitements par anoxie dynamique. En outre, une durée de cinq jours, correspondant à une semaine ouvrée, est suffisante pour détruire des larves et des œufs de capricorne avec un taux de mortalité de $100 \%$.

Le deuxième constat important de ces travaux est qu'un traitement à basse pression respecte a priori les polychromies des objets si l'humidité dans l'enceinte est régulée pendant toute la durée du traitement. Dans ces conditions, les basses pressions apparaissent sans danger pour les polychromies. Cette affirmation doit être validée par rapport à des polychromies plus anciennes, donc plus fragiles. Suite à ces résultats prometteurs, ARC-Nucléart souhaite désormais proposer le traitement de désinsectisation à basse pression en tant que procédé alternatif à l'irradiation gamma, notamment pour des objets associant des matériaux sensibles aux rayonnements ionisants tels que des matériaux transparents. Bien que l'ensemble du travail expérimental ait été réalisé sur des échantillons en bois, il nous semble intéressant d'élargir notre champ d'étude aux matériaux organiques encore plus poreux que le bois comme le textile, le papier, le cuir, les poils et les plumes, pour lesquels la diffusion de l'oxygène et de la vapeur d'eau sera favorisée.

Malgré les bons résultats obtenus sur les insectes, nous rappelons toutefois les limites intangibles de ce procédé de désinsectisation, communes avec l'anoxie ou l'irradiation gamma : le traitement est purement curatif et en aucun cas préventif. De plus, contrairement à l'irradiation gamma selon la dose, le procédé n'est pas efficace pour la désinfection, c'est-à-dire la destruction des micro-organismes (bactéries, champignons ou levures). En outre, il reste encore à déterminer dans une étude complémentaire les mécanismes exacts qui contrôlent la destruction des insectes en répondant aux questions suivantes : est-ce le manque d'oxygène ou la basse pression qui permet aux stigmates de l'insecte de rester plus longtemps ouvert ? Si, à la place de l'azote humidifié, il est envoyé uniquement de l'air humide avec de l'oxygène, l'effet létal serait-il aussi efficace? Si la réponse à cette dernière question est positive, il serait possible alors de simplifier le dispositif en faisant l'économie d'un balayage d'azote.

Deux axes d'amélioration du protocole ont été identifiés pour permettre une large diffusion de ce nouveau procédé de désinsectisation auprès des professionnels de la conservation :

- il est possible de rendre les insectes plus sensibles au traitement en les plaçant dans des conditions de température pour lesquelles leur activité métabolique est maximale (de 25 à $30^{\circ} \mathrm{C}$ ). À ces températures, leur mécanisme de défense contre le manque d'oxygène et le dessèchement pourrait se révéler moins performant du fait des besoins en eau et en oxygène plus importants ${ }^{3}$. En augmentant la température, il devrait alors être possible de réduire davantage le temps du traitement en vérifiant qu'il n'y ait pas d'impact de la température sur la polychromie ;

- bien que l'enceinte d'ARC-Nucléart possède une capacité de traitement relativement importante $\left(2,8 \mathrm{~m}^{3}\right)$, celle-ci est insuffisante pour des objets volumineux. Toutefois, il est à noter qu'il existe dans le commerce des enceintes de très grandes dimensions $\left(30 \mathrm{~m}^{3}\right)$ en matériaux plastiques, capables de tenir mécaniquement au vide, et utilisées pour la désinsectisation des graines. Il deviendrait alors envisageable d'effectuer des désinsectisations par ce procédé sur des volumes du même ordre que ceux pratiqués en anoxie dynamique dans des poches.

\section{Notes}

1. Ramière, 2002, p. 291-302. Gunn, 2008, p. 15-22. Gunn, 2006, publication électronique. Valentin, 1993, p. 263-278.

2. Maurin, 2007 , p. 90

3. Dajoz, 2010, p. 27-29.

\section{Bibliographie}

Dajoz R., 2010, Dictionnaire d'entomologie, anatomie, systématique, biologie, édition Lavoisier Tec \& Doc.

Gunn M., 2008 (janvier-février), "Désinsectiser les collections ", La Lettre de l'OCIM, $\mathrm{n}^{\circ} 115$, p. 15-22.

Gunn M., H. Ziaeepour, F. Merizzi, C. Naffah, "Anoxia - treatment by oxygen deprivation: optimizing treatment time of museum objects", arXiv:physics/0611199 [physics.chem-ph], http://arxiv.org/ftp/physics/ papers/0611/0611199.pdf

Maurin E., Lyon F., 2007, «Impact de la congélation sur les propriétés mécaniques des assemblages de bois collés. Application à la désinsectisation du mobilier par congélation ", Actes de la Journée d'étude Conserver aujourd'hui, p. 90.

Ramière R., 2002, « La désinfection des biens culturels par irradiation gamma ", dans Les contaminants biologiques des biens culturels, éditions Elsevier/M. F. Roquebert, Paris, p. 291302.

Valentin N., 1993, "Comparative analysis of insect control by nitrogen, argon and carbon dioxide in museum, archive and herbium collections", International Biodeterioration and Biodegradation, $\mathrm{n}^{\circ} 32$, p. 263-278. 